\title{
Surveillance of Aedes aegypti (L.) Mosquitoes in Mumbai International Seaport (India) to Monitor Potential Global Health Risks
}

\author{
Kaushal Kumar, ${ }^{1}$ Abhay Kumar Sharma, ${ }^{1}$ Manas Sarkar, ${ }^{1}$ \\ Arun Chauhan, ${ }^{1}$ and Rajeev Sharma ${ }^{2}$ \\ ${ }^{1}$ Centre for Medical Entomology \& Vector Management, National Centre for Disease Control, 22 Sham Nath Marg, Delhi 110054, India \\ ${ }^{2}$ Global Disease Detection, Indian Centre, National Centre for Disease Control, 22 Sham Nath Marg, Delhi 110054, India
}

Correspondence should be addressed to Abhay Kumar Sharma; drabhayncdc@gmail.com

Received 13 February 2014; Revised 13 May 2014; Accepted 4 June 2014; Published 29 June 2014

Academic Editor: José A. Martinez-Ibarra

Copyright (C) 2014 Kaushal Kumar et al. This is an open access article distributed under the Creative Commons Attribution License, which permits unrestricted use, distribution, and reproduction in any medium, provided the original work is properly cited.

\begin{abstract}
Aedes mosquitoes are highly invasive and can survive almost any climatic conditions. They transmit a number of major world's deadly diseases. Therefore, a study was undertaken during December 2010 to evaluate the entomo-epidemiological risk of Aedes mosquito borne diseases (VBD) in Mumbai international seaport areas to minimize potential global health risks and prevent introduction of new VBD in India. Surveys were undertaken in operational and residential areas of Mumbai Port Trust (MPT). All the entomological indices were found to be above the critical level, prescribed for seaports by International Health Regulations Act, 2005. The operational areas where large goods are handled from cargo ships were found to be more prone to mosquito breeding comparing to residential areas. High insecticide tolerance of Aedes aegypti population against temephos and fenthion from Mumbai port area is reported for the first time. A careful and regular invigilation of the international seaports to prevent building up of vector density of dengue/chikungunya and yellow fever is recommended.
\end{abstract}

\section{Introduction}

Aedes is a genus of mosquitoes originally found in tropical and subtropical zones. It is considered highly invasive in nature and can carry a variety of pathogens that can be transmitted to humans. The species Aedes aegypti L. and Aedes albopictus (Skuse) are the primary vectors of concern worldwide. Ae. aegypti mosquito is the main vector that transmits the viruses that cause dengue. It is also known to transmit filarial infections of Wuchereria bancrofti and Dirofilaria immitis and avian parasite Plasmodium gallinaceum [1]. Ae. albopictus is primarily a forest species that has become adapted to rural, suburban, and urban human environments. These species commonly transmit dengue virus (DENV) around the globe; an estimated 50-100 million new dengue fever infections occur each year, causing $~ 500,000$ cases of dengue hemorrhagic fever (DHF) and $>20,000$ deaths [2-5]. It can also transmit yellow fever, chikungunya, and ross river viruses.
The incidences of vector-borne diseases are increasing alarmingly due to many factors including uncontrolled urban developments that support breeding of vector mosquitoes. World Health Organization (WHO) in 2010 stratified the current situation of dengue/DHF in India under category A, which means a major public health problem, leading cause of hospitalization and death among children. In India, National Vector Borne Disease Control Programme (NVBDCP) reported 28,055 dengue cases in 2010 from 31 out of 35 states in India (highest ever in a year) [6]. In 2013, a total of 22,092 dengue cases and 74 deaths were reported form the country (of which 23 deaths were from Maharashtra only) [6]. In Maharashtra state sporadic cases of dengue were reported in 1973, 1983, and 2000. Morbidity in Barsi, Maharashtra (1973), was about $37.5 \%$. Recently the cases of Chikungunya are reported from villages Mungi, Balamtakli, and Madhi (district Ahamadnagar), Malegaon city (district Nashik), and all 8 districts of Marathwada region and in Vidharbh region 7 districts: Akola, Washim, Buldhana, Yeotmal, Nagpur, Wardha, 
and Chandrapur [7]. Mumbai, too, had recorded 116, 416, 1008, and 250 dengue cases in 2010, 2011, 2012, and 2013, respectively [8]. Moreover, India shares sea route/connection with majority of yellow fever (YF) endemic countries. This raises more concern in India to control Aedes mosquito breeding in seaport areas to prevent any introduction of YF virus in the country. As per International Health Regulations Act, 2005 [9], all the international airports/seaports, ground crossings, and peripheral areas up to 400 meters should be kept free from Aedes mosquito breeding or indices should be kept less than one to eliminate the chance of spreading disease or vectors to any part of the world. Therefore, in view of the endemic situation in Maharashtra and as a member country of WHO, an entomo-epidemiological survey was undertaken to know the present scenario of entomological indices, presence of DENV in Aedes mosquitoes, insecticide susceptibility status, and so forth in and around Mumbai port area to assess potential global health risks.

\section{Materials and Methods}

2.1. Study Area. Present entomological surveillance was undertaken in and around the Mumbai seaport in December 2010. Mumbai the capital of Maharashtra states is the second most populated city in India. It is located towards west side of the Indian seacoast at $18^{\circ} 58^{\prime} \mathrm{N}$ and $72^{\circ} 50^{\prime} \mathrm{E}$. Mumbai weather never gets "cold" since there is no real winter in the place. In fact when the minimum temperature goes below $15^{\circ} \mathrm{C}$, it is considered "chilly." Most of the year the weather is hot and humid throughout the day and night. In December, humidity is low and the day highs hover around $32.0^{\circ} \mathrm{C}$ and nights do not fall below $18^{\circ} \mathrm{C}$. Mumbai seaport has been the principal gateway to India and playing a pivotal role in the commercial shipping and connections with the major cities of different countries. Statutory autonomous corporation known as Mumbai Port Trust administrates Mumbai port. MPT is a major historical port established in 1873. This port is fully integrated multipurpose port handling container, dry bulk, liquid bulk, and break bulk cargos. During the present investigation, mosquito survey was undertaken in and around the seaport areas covering a perimeter of $500 \mathrm{~m}$ around operational areas of Mumbai seaport that includes three docks: Indira Dock, Victoria Dock, and Prince's Dock which comprises the administrative block, canteen sheds, and so forth. Aedes survey was also carried out into two residential colonies: Parichya colony and Carnac Bunder colony. Nonresidential Butcher Island situated at a distance of $5 \mathrm{Km}$ from seashore was also included in this study. Butcher Island has been included as premises under operational area for data analysis. In seaport, baygon and pyrethrum are being used for spraying against adult and temephos granules for larvae as vector control measures.

2.2. Entomological Surveillance. Entomological surveillance of Aedes mosquito has been standardized on different indices based on the simple determination of presence or absence of Aedes larvae either in each container or somewhat in each house $[10,11]$. The house index (HI, percentage of houses positive for larvae), container index (CI, percentage of containers positive for larvae), Breteau index (BI, number of positive containers per 100 houses), and Premises Index were calculated. All nonhermetically closed containers containing any volume of water were considered as potential breeding sites. All water-holding containers were examined. Either the name/type of the containers (namely, tire, cooler, fire extinguisher bucket, etc.) or construction materials (namely, cement tank, earthen pots, and plastic container) classified the breeding sites. The number of surveyed premises, houses, positive containers (with Aedes pupae or larvae), and houses with $\geq 1$ positive container has been recorded in the study.

2.3. Detection of Dengue Virus (DENV) in Mosquitoes. Larvae and adults were collected from the MPT areas and brought to the Centre for Medical Entomology and Vector Management (National Centre for Disease Control, Delhi) for detection of DENV inside mosquitoes by antigen-capture enzyme linked immunosorbent assay (ELISA) followed by the protocol described by Gajanana [12]. Wild caught adult mosquitoes and adults reared from larvae were tested separately. Sex wise pool of 3-10 unfed Ae. aegypti mosquitoes were prepared and tested to see the presence of DENV. Mono-4 clonalantibody (MAb), D14G2 (1:1000), as capture antibody (broadly reactive against four serotypes of DENV) and Mab-peroxidase conjugate MAb 6B6C-1 as detector antibody (1:2000) were used. The ELISA plate contained known DENV infected suckling mouse brain homogenate as positive control and the homogenate of uninfected adult Ae. aegypti as negative controls. Mean [ \pm 4 standard deviation (SD)] optical density (OD) of the normal laboratory reared mosquito pools (negative control) was taken as threshold level and any divergence of $\mathrm{OD}$ of the tested mosquito population that crosses this threshold level is considered as positive for DENV infection.

2.4. Insecticide Susceptibility Assays. Larvae were collected and brought to the laboratory to determine the susceptibility status. Multiple concentration tests were conducted in order to establish baseline data of susceptibility and resistance. Groups of 25 third and early fourth instar larvae of F1 were tested with different concentrations of temephos (dose $0.012 \mathrm{mg} / \mathrm{L}$ ) and fenthion (dose $0.025 \mathrm{mg} / \mathrm{L}$ ) in $250 \mathrm{~mL}$ of water with control having no insecticide solution but with acetone. Each bioassay tested a minimum of six concentrations with at least three replicates per concentration [13]. Larval mortality was recorded after 24 hours and lethal concentration (LC) values were calculated using dosagemortality regression probit analysis [14].

2.5. Statistical Analysis. Kolmogorov-Smirnov (with DallalWilkinson-Lilliefor $P$ value), D’Agostino and Pearson Omnibus and Shapiro-Wilk normality test have been performed to see whether the data follows a Gaussian distribution. Appropriate statistical tests was implemented to calculated the corresponding $P$ values. Larval survey data of different breeding sites based on the name/type of the containers (namely, tire, cooler, fire extinguisher bucket, etc.), construction materials (namely, tin, cement tank, clay pots, and plastic container) 


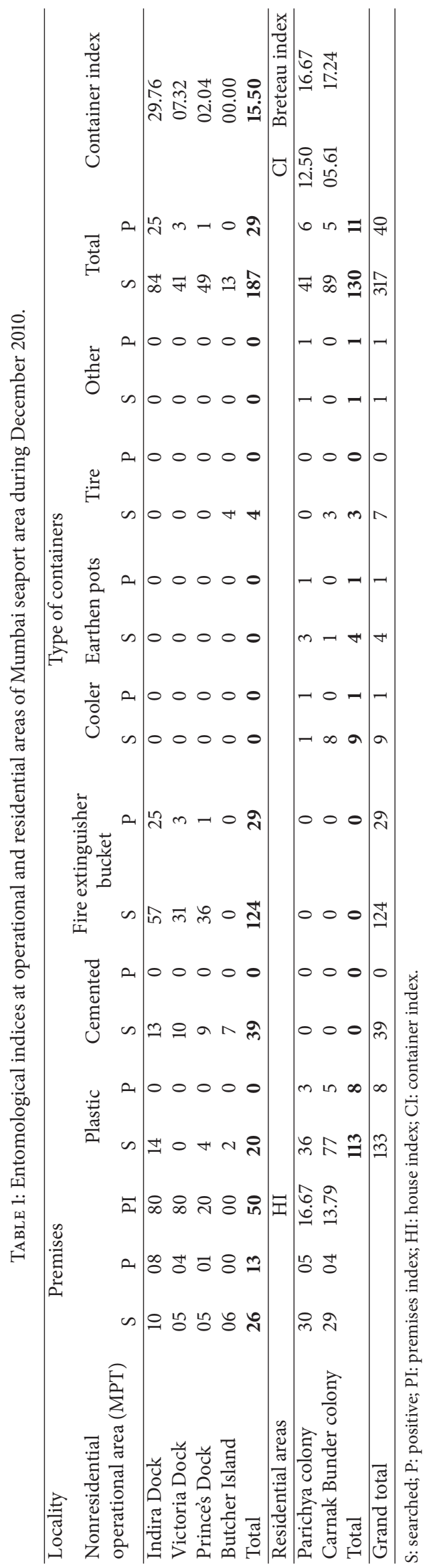


TABLE 2: Probability distribution showing positive mosquito (larvae) breeding among different types of containers at Mumbai seaport area during December 2010.

\begin{tabular}{|c|c|c|c|c|c|c|c|c|}
\hline & Plastic & Cemented & Fire extinguisher bucket & Cooler & Earthen pot & Tire & Other & Total \\
\hline \multicolumn{9}{|c|}{ Operational area (MPT) } \\
\hline Indira Dock & $0 \%$ & $0 \%$ & $44 \%$ & $0 \%$ & $0 \%$ & $0 \%$ & $0 \%$ & $30 \%$ \\
\hline Victoria & $0 \%$ & $0 \%$ & $10 \%$ & $0 \%$ & $0 \%$ & $0 \%$ & $0 \%$ & $7 \%$ \\
\hline \multicolumn{9}{|l|}{ Dock } \\
\hline Prince's & $0 \%$ & $0 \%$ & $3 \%$ & $0 \%$ & $0 \%$ & $0 \%$ & $0 \%$ & $2 \%$ \\
\hline \multicolumn{9}{|l|}{ Dock } \\
\hline Total & $0 \%$ & $0 \%$ & $23 \%$ & $\mathbf{0 \%}$ & $0 \%$ & $\mathbf{0 \%}$ & $\mathbf{0} \%$ & $16 \%$ \\
\hline \multicolumn{9}{|l|}{ Residential areas } \\
\hline $\begin{array}{l}\text { Parichya } \\
\text { colony }\end{array}$ & $8 \%$ & $0 \%$ & $0 \%$ & $100 \%$ & $33 \%$ & $0 \%$ & $100 \%$ & $15 \%$ \\
\hline Carnak & $6 \%$ & $0 \%$ & $0 \%$ & $0 \%$ & $0 \%$ & $0 \%$ & $0 \%$ & $6 \%$ \\
\hline \multicolumn{9}{|l|}{$\begin{array}{l}\text { Bunder } \\
\text { colony }\end{array}$} \\
\hline Total & $7 \%$ & $\mathbf{0 \%}$ & $\mathbf{0 \%}$ & $11 \%$ & $25 \%$ & $\mathbf{0 \%}$ & $100 \%$ & $8 \%$ \\
\hline Grand total & $6 \%$ & $0 \%$ & $23 \%$ & $11 \%$ & $25 \%$ & $0 \%$ & $100 \%$ & $13 \%$ \\
\hline
\end{tabular}

TABle 3: Insecticide susceptibility status of Ae. aegypti mosquitoes collected from Mumbai seaport area during December 2010.

\begin{tabular}{|c|c|c|c|c|c|c|c|}
\hline Insecticides & $\mathrm{LC}_{50}$ & $95 \%$ CI & $\mathrm{LC}_{90}$ & $95 \% \mathrm{CI}$ & $\mathrm{Chi}^{2}(\mathrm{DF})$ & Slope & Slope " $t$ " (DF) \\
\hline \multirow{2}{*}{ Temephos } & \multirow{2}{*}{0.029} & \multirow{2}{*}{$0.017-0.049$} & \multirow{2}{*}{0.107} & \multirow{2}{*}{$0.062-0.189$} & $13.983(3)$ & \multirow{2}{*}{2.27} & $7.069(3)$ \\
\hline & & & & & $P=0.0029$ & & $P=0.0058$ \\
\hline \multirow{2}{*}{ Fenthion } & \multirow{2}{*}{0.018} & \multirow{2}{*}{$0.011-0.032$} & \multirow{2}{*}{0.043} & \multirow{2}{*}{$0.025-0.076$} & $15.755(3)$ & \multirow{2}{*}{3.44} & $4.72(3)$ \\
\hline & & & & & $P=0.0013$ & & $P=0.018$ \\
\hline
\end{tabular}

DF: degree of freedom; LC: lethal concentration; CI: confidence interval.

was analyzed. Plain vanilla probabilistic and chi-square frequency tests to judge the significance of chance of getting positive containers (i.e., containers contain single Aedes larvae) in different areas were applied. For all areas different entomological indices (namely, HI, CI, BI, and/or Premises Index) were calculated.

\section{Results}

3.1. Entomological Surveillance. In operational areas of MPT, out of the total 26 premises, 13 were found positive for Ae. aegypti breeding giving an overall premises index of $50 \%$ with high CI in Indira Dock (29.76\%); however, no breeding in Butcher Island could be found at this time (Table 1).

Among residential colonies, Parichya colony showed higher HI and CI (16.7\% and 12.5\%, resp.) than Carnak Bunder colony, where BI (17.24) was relatively high (Table 1). The most of the breeding was detected in fire extinguisher bucket kept in the different sheds along with other containers. Various breeding containers searches were tires, coolers, tin containers, cement tanks, earthen pots, fire extinguisher bucket, and others. Distribution of mosquito breeding in different containers in nonresidential operational areas of MPT and two residential colonies adjacent to the seaport are shown in Table 1. Among these different containers, fire extinguisher bucket shows only breeding sites in operational areas with overall probability of $23 \%$, whereas in residential areas coolers, earthen pots, and other containers (mainly disposable plastic cups and glass) show maximum potential for breeding based on plain vanilla probability model (Table 2 ). No other genus of mosquito larvae was found during the surveillance.

3.2. Detection of Dengue Virus (DENV) in Mosquitoes. A total of 331 Ae. aegypti mosquitoes ( 99 males and 232 females) were tested for DENV infection, out of which 250 samples were reared from larvae collected from MPT and the rest were adult caught wild mosquitoes. However, all samples were found negative for DENV.

3.3. Insecticide Susceptibility Assays. Results of insecticide susceptibility assays are presented in Table 3 . Baseline assay shows that LC90 (0.107) against temephos is quite higher than the WHO recommended diagnostic dose. Overall, population was found to have high tolerance against both temephos and fenthion.

3.4. Statistical Analysis. Chi-square frequency test was used to understand whether any statistical dependency is present among breeding positivity in two types of areas (i.e., operational and residential) surveyed. Here, we found that operational areas of MPT are more prone to mosquito breeding comparing to residential areas (Chi-square statistics $=29.9$, 
degree of freedom $=6$, alpha level $=0.05$, and $\chi^{2}=12.6$; Chisquare statistics $>\chi^{2}$ ). We further analyzed that there is no significant dependency between types of positive container for breeding in two residential colonies (Chi-square statistics $=3.0$, degree of freedom $=4$, alpha level $=0.05$, and $\chi^{2}=9.5$; Chi-square statistics $<\chi^{2}$ ).

\section{Discussion}

Vector borne diseases are on the rise in last few years with epidemics reported in many countries. In the present study, it was assessed how different types of containers contribute to the breeding of Ae. aegypti and how all this knowledge may help in establishing better control strategy for Ae. aegypti mosquitoes in MPT as well as other seaports in India. During investigation it was observed that MPT having limited infrastructural facilities and inadequate trained workforce to control the mosquitoes may be one of the factors for establishment and expansion of Aedes mosquito vectors in peak seasons.

During our survey, the highest percentage of positive containers for larvae has been shown in fire extinguisher buckets in operational areas (Table 1). Interestingly, these positive containers (fire extinguisher buckets) are emergency instrument and technically it should always be filled with water. However, the stored water should be changed regularly. Unfortunately, our findings revealed that the poor management of these buckets is the main cause for mosquito breeding. It is worth noting that in some areas we found that $100 \%$ of surveyed containers are positive for mosquito breeding.

We could not detect any mosquito sample positive with DENV, but this was perhaps due to the low viral load in the month of December (nonpeak season). However, we found that all the entomological indices are above the critical level, as prescribed in International Health Regulations (2005) [9]. The earlier studies conducted in seaport in India also showed high entomological indices and even during the dry months $[15,16]$. Therefore, it is quite clear that seaport authorities need to give more attention to implementing International Health Regulations (2005) to counter the mosquito-breeding problem. Mosquito breeding surrounding seaport and/or international border is not just a simple local health problem or biting nuisance; it is a serious threat to global health security. Thus, a careful invigilation of the international airports and seaports by the trained scientists/vector control personnel is recommend to prevent breeding and unwanted export of vector species and identify any accidental and/or deliberate import of alien arthropod vectors as biosecurity and quarantine measures to prevent potential international health risks. Moreover, future research priorities should include regular surveillance of arthropod vectors, as well as molecular diagnostics of diseases, identification of vectors, and phylogenetic origin of the vector strains in seaport areas. A rapid action team of trained scientist and health workers, equipped with conventional and modern scientific instrumentations and suitable vector control tools, would be able to counter vector breeding during any epidemics and outbreaks situation.

\section{Conflict of Interests}

The authors do not have any conflict of interests.

\section{References}

[1] R. C. Russell, C. E. Webb, and N. Davies, “Aedes aegypti (L.) and Aedes polynesiensis marks (Diptera: Culicidae) in Moorea, French Polynesia: a study of adult population structures and pathogen (Wuchereria bancrofti and Dirofilaria immitis) infection rates to indicate regional and seasonal epidemiological risk for dengue and filariasis," Journal of Medical Entomology, vol. 42, no. 6, pp. 1045-1056, 2005.

[2] D. J. Gubler, "Human arbovirus infections worldwide," Annals of the New York Academy of Sciences, vol. 951, pp. 13-24, 2001.

[3] D. J. Gubler, "Epidemic dengue/dengue hemorrhagic fever as a public health, social and economic problem in the 21st century," Trends in Microbiology, vol. 10, no. 2, pp. 100-103, 2002.

[4] D. J. Gubler, "The changing epidemiology of yellow fever and dengue, 1900 to 2003: full circle?" Comparative Immunology, Microbiology and Infectious Diseases, vol. 27, no. 5, pp. 319-330, 2004.

[5] S. Bhatt, P. W. Gething, O. J. Brady et al., "The global distribution and burden of dengue," Nature, vol. 496, no. 7446, pp. 504-507, 2013.

[6] "National Vector Borne Disease Control Programme (NVBDCP)," 2012, http://nvbdcp.gov.in/den-cd.html.

[7] The Public Health Department, Government of Maharashtra, http://www.maha-arogya.gov.in/diseasesinfo/Dengue/default .htm.

[8] S. D. Roy, "Virulent dengue strain at work, 250 cases already reported in Mumbai," 2013, http://timesofindia.indiatimes .com/city/mumbai/Virulent-dengue-strain-at-work-250\%20casesalready-reported-in-Mumbai/articleshow/20937248.cms.

[9] WHO, International Health Regulations 2005, World Health Organization, 2nd edition, 2008, http://www.who.int/csr/ihr/ IHR_2005_en.pdf.

[10] W. Tun-Lin, B. H. Kay, A. Barnes, and S. Forsyth, "Critical examination of Aedes aegypti indices: correlations with abundance," The American Journal of Tropical Medicine and Hygiene, vol. 54, no. 5, pp. 543-547, 1996.

[11] D. A. Focks, A Review of Entomological Sampling Methods and Indicators for Dengue Vectors, World Health Organization, Geneva, Switzerland, 2003.

[12] A. Gajanana, R. Rajendran, V. Thenmozhi, P. P. Samuel, T. F. Tsai, and R. Reuben, "Comparative evaluation of bioassay and ELISA for detection of Japanese encephalitis virus in field collected mosquitos," The Southeast Asian Journal of Tropical Medicine and Public Health, vol. 26, no. 1, pp. 91-97, 1995.

[13] World Health Organization, "Instructions for determining the susceptibility or resistance of mosquito larvae to insecticides," Tech. Rep. WHO/VBC/81.807, 1981.

[14] D. J. Finney, Probit Analysis, Cambridge University Press, Cambridge, UK, 3rd edition, 1971.

[15] K. S. Gill, S. K. Sharma, R. Katyal, and K. Kumar, “Aedes aegypti survey of Chennai seaport/airport, India," Dengue Bulletin, vol. 24, pp. 121-123, 2000.

[16] S. N. Sharma, S. Kumar, B. P. Das et al., "Entomological indices of Aedes aegypti at some international airports and seaports of southern India-a report," Journal of Communicable Diseases, vol. 37, no. 3, pp. 173-181, 2005. 

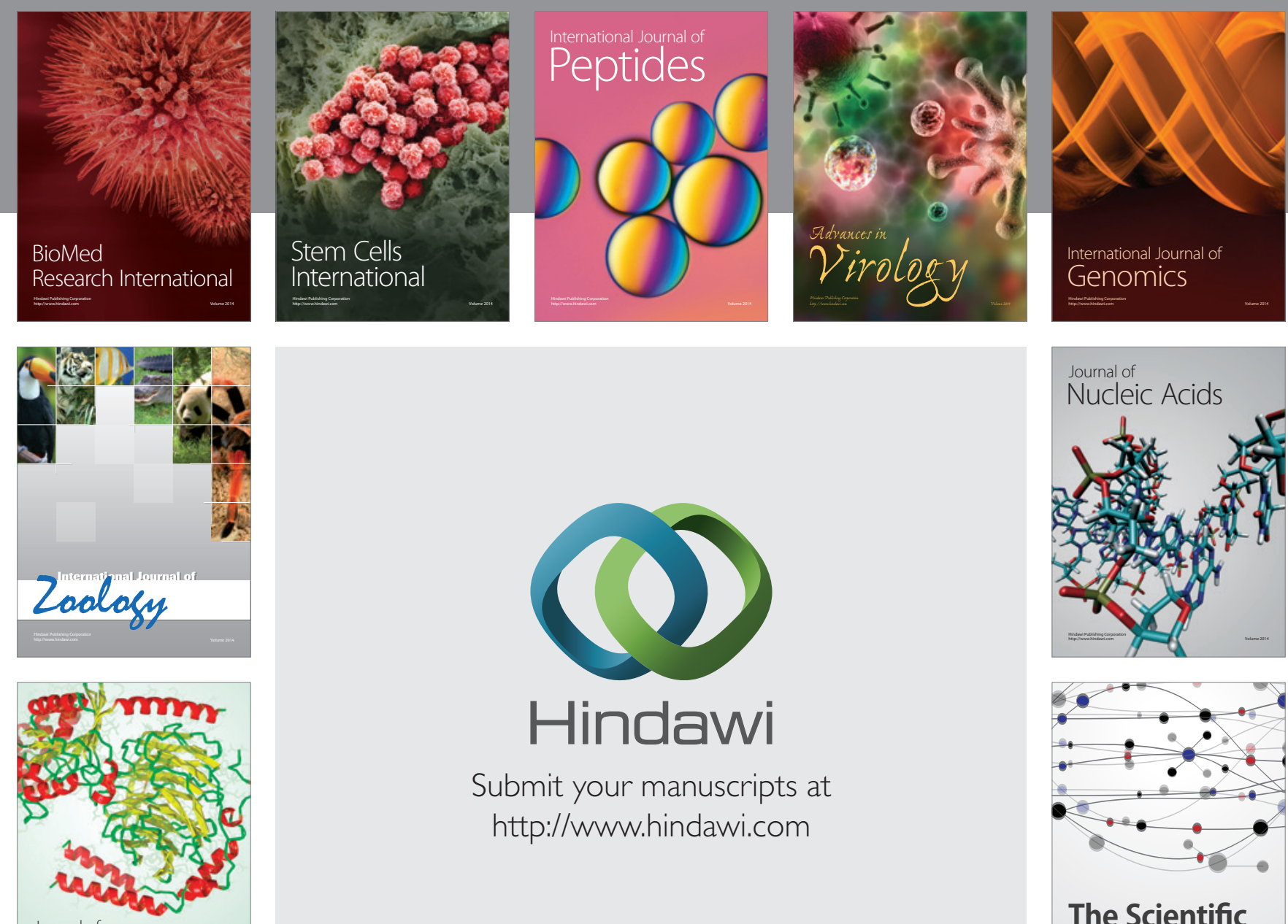

Submit your manuscripts at

http://www.hindawi.com

Journal of
Signal Transduction
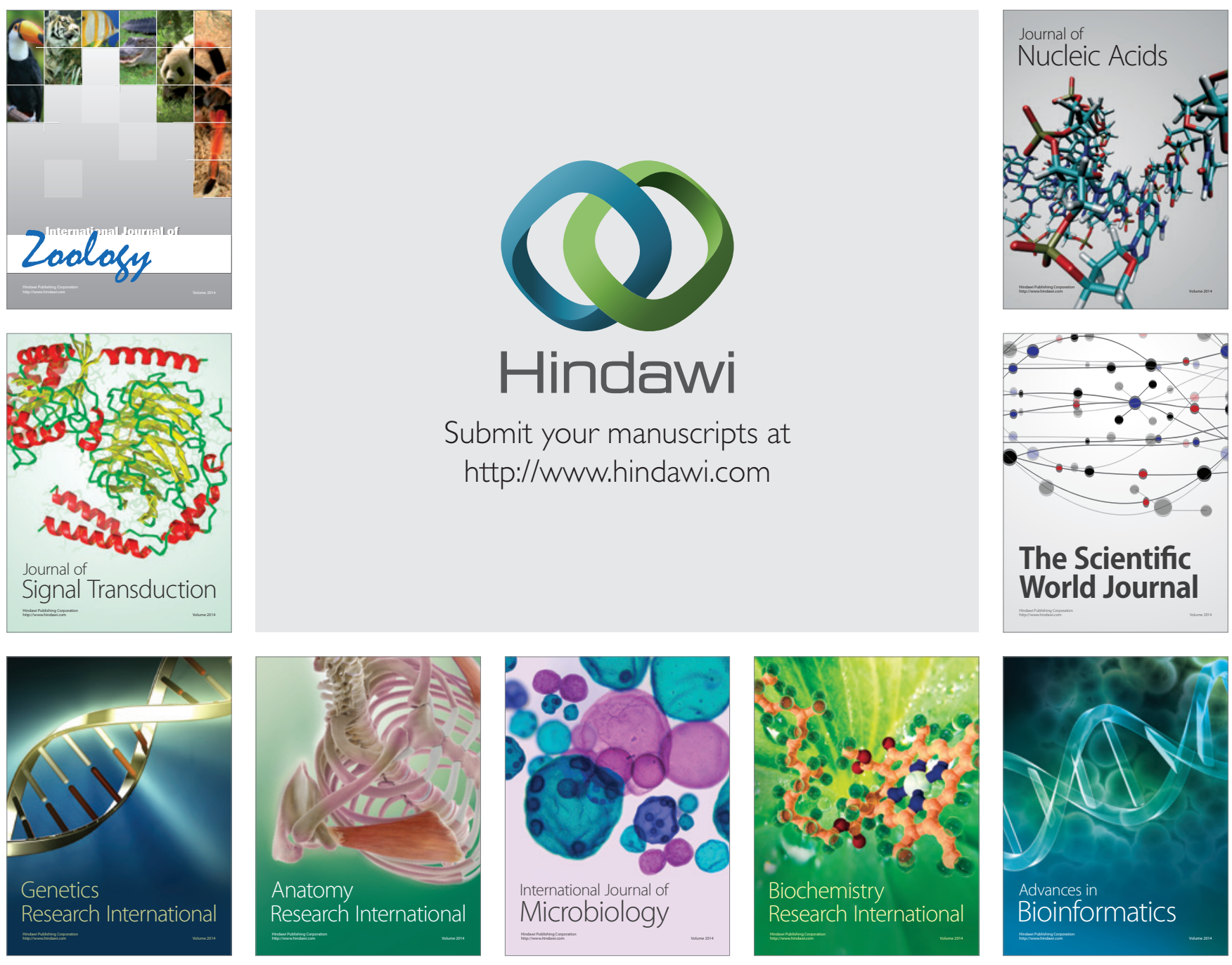

The Scientific World Journal
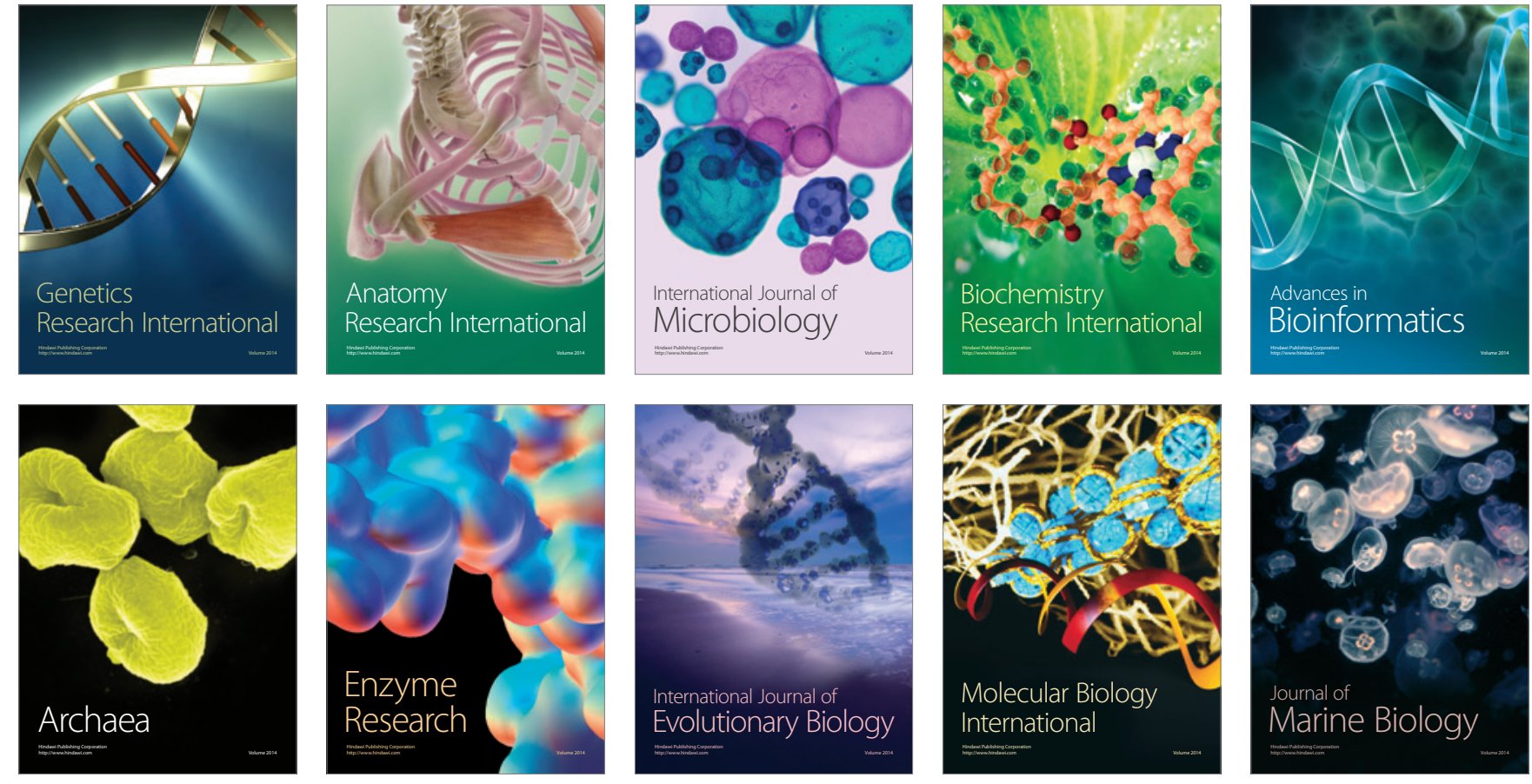